\title{
Modeling of Duck Density and Complex Stocking Time in Rice-Duck Agroecosystems in Terms of Economic and Ecological Benefits
}

\author{
Dahong Xiong, ${ }^{1}$ Kui Fang, ${ }^{1}$ Ying Luo, ${ }^{2}$ and Xiaopeng Dai ${ }^{1}$ \\ ${ }^{1}$ College of Information Technology, Hunan Agricultural University, Changsha 410128, China \\ ${ }^{2}$ Center of Information and Networks, Hunan Agricultural University, Changsha 410128, China \\ Correspondence should be addressed to Kui Fang; boshi863@163.com
}

Received 1 September 2013; Accepted 4 December 2013; Published 20 March 2014

Academic Editor: Shuping He

Copyright ( 92014 Dahong Xiong et al. This is an open access article distributed under the Creative Commons Attribution License, which permits unrestricted use, distribution, and reproduction in any medium, provided the original work is properly cited.

Rice-duck integrated farming is an effective step under today's sustainable development background. To make better economic and ecological benefits, a rice-duck agroecosystem is established and kept, in which the paddy field, rice, and the duck mutually promote one another. But the duck density and complex stocking time must be rationally selected. Aiming to attain quantitative assessment and optimal selection of the duck density and complex stocking time in this kind of systems, a methodology based on proposed mathematical models in terms of comparative economic and ecological benefits is addressed. Then the models are solved by a hybrid intelligent algorithm NN-GA that integrates the Neural Networks (NN) and Genetic Algorithm (GA), making use of the fitting ability in nonlinear fitness context of Neural Networks and the optimization ability of the Genetic Algorithm. Besides, numerical examples are demonstrated in order to test the proposed models. Results reveal that the methodology is reasonable and feasible.

\section{Introduction}

Nowadays, the sustainable development has been a hotspot issue and there is a growing concern on high crop production because of its high cost of pesticides and expensive fertilizers and on their corresponding forms of environmental pollution [1]. Besides, due to rice cultivation, greenhouse gases are emitted from the rice field as by-products, including $\mathrm{CH}_{4}$, $\mathrm{CO}_{2}$, and $\mathrm{N}_{2} \mathrm{O}$ [2]. Rice-duck integrated farming is such an effective way that an agroecosystem is established and kept to reduce the dosage of pesticides, herbicides, and chemical fertilizers, to reduce the fluxes of greenhouse gas emission and to improve the quality of rice and duck products. In a rice-duck integrated ecosystem, the paddy field together with rice provides ducks with an ideal growing environment, protecting them from strong sunshine or heavy rain. While ducks help, through their activities in the paddy field, to interrow and fertilize the paddy field soil, to ecologically control rice diseases and pests, and to reduce the population of weeds and the emission of methane and nitrous oxide.
Many researchers have discussed the issue to reveal the potential significance of rice-duck integrated farming [35]. For example, Qin et al. proposed a methodology of population control modeling of cnaphalocrocis medinalis guenee in rice-duck integrated farming systems [6]. However, how many ducks should be bred in a given paddy field? How long should they grow together with rice in the paddy field? What can be done for a farmer to select a rational duck density and a reasonable complex cultivation and stocking span of time? That is one of the most important problems in practice. Jin et al. have investigated the effect of raising ducks in paddy field at a density of 180 ducks $/ \mathrm{hm}^{2}$ [7]. And there exists no literature contribution on the issue of integrated effect of duck density and complex cultivation and stocking time (called complex stocking time for short next) on economic and ecological benefits that can be attained.

In fact, the selection of duck density together with the complex stocking time for a rice-duck ecosystem may suffer from nonlinear relations between duck density and the net 
farming income and ecological benefits. Some factors are also characterized with uncertainty and not easy to describe and represent clearly. For example, the cost of the ducks stocking, which is essential for the decision of the duck density, is uncertain as the stochastic death rate is affected and constrained by the surroundings around the ecosystem and other factors, such as the climate, possible epidemics, and so on. As a result, the selection of the duck density and complex growing time is really complicated. Some scholars in related fields have discussed similar analysis and management problems [8-12]. For example, Kim et al. [13] proposed their optimal design and global sensitivity analysis of biomass supply chain networks for biofuels under high level of uncertainty in supply amounts, market demands, market prices, and processing technologies. Chami et al. [14] have discussed the impacts of combined technical and economic measures on water saving in agriculture based on a mathematical stochastic model able to represent uncertainty in water availability. Sun et al. [15] analyzed the uncertainty for the evaluation of agricultural soil quality based on digital soil maps. Haro et al. [16] proposed a method of stochastic hydroeconomic modeling for optimal management of agricultural groundwater nitrate pollution under the circumstance of hydraulic conductivity uncertainty. In a word, the problem can be solved based on some models according to the domain knowledge, experiments, and some statistical rules and estimates [17-19].

This paper discusses the quantitative assessment and modeling of the duck density and complex stocking time in the rice-duck ecosystem in terms of economic and ecological benefits, addressing a methodology to select an optimal pair of duck density and complex stocking time based on the proposed mathematical models, which are solved by a hybrid intelligent algorithm $N N-G A$ that integrates Neural Networks (NN) and Genetic Algorithm (GA), taking advantage of the fitting ability in nonlinear fitness context of $\mathrm{NN}$ and the optimization ability of GA. The rest of paper is organized as follows. Section 2 proposes the method and introduces the NN-GA algorithm after the problem statement. Section 3 presents numerical examples and analyzes the results to test the effectiveness of the proposed method. Finally, Section 4 concludes this paper and describes future research progress directions.

\section{Method and Algorithm}

2.1. Problem Statement. Rice and ducks in the rice-duck integrated ecosystem can mutually promote their growth on condition that the duck density is of a rational size and that the complex stocking time is reasonable. According to agricultural knowledge and experience, given other natural conditions, means, and measures of management being the same in a rice-duck integrated farming ecosystem as those in a paddy system without duck complex stocking, the comparative benefits are decided mainly by two factors: one is the duck density, the other is the start and end time of duck complex stocking process in the paddy field. Raising too many ducks or the complex stocking time is too long in a certain paddy field can badly affect rice tillering, reduce the highest seedlings and efficient panicles, and even affect the rice with respect to panicle and maturity, which means a considerable reduction of rice yields. In addition, because there are not enough pests or weeds to feed such a large amount of ducks, some rice leaf may be their food inevitably. However, raising too few ducks or with too short a complex time prevents the potential economic and ecological returns being attained. Therefore, planning models are essential to make a reasonable selection of the duck density and complex stocking time.

In order to establish planning models, some assumptions and related concepts are given next.

\subsection{Assumptions}

(1) Select the traditional means of rice planting without duck complex stocking and growing together in the paddy field as the reference situation to make a better comparison, given the rest conditions being the same. We call the reference standard CKO in this paper if no confusion arises, while we name a rice-duck integrated agroecosystem CK for short.

(2) Given the basic costs and outcome of CK and CK0 being the same, such as the cost of rice seeds and small farming equipments depreciation, financial supporting from the intergovernmental transfer system, rent and management costs of paddy fields. We neglect these parts when calculating and comparing the costs or outcome of $\mathrm{CK}$ and CK0, so the comparative benefits, namely, the extra economic and ecological benefits in CK compared with CK0, can be calculated.

(3) Suppose the starting point of the complex stocking is 7 to 10 days after the rice's transplantation when it turns green and the ducks are generally 15 to 20 days old, that is to say, it is from this time-point on that the riceduck complex agroecosystem is supposed to work. That is due to two facts: firstly, if stocking ducks in the rice field complexly with rice before it turns green, it will be greatly influenced by ducks; secondly, a little duck cannot grow into a service duck until it is 15 to 20 days old, for it ought to be trained to capture food and peck mud first. Consider mainly the effects of duck density $d$ and complex stocking time $t$ in paddy field when calculating the comparative benefits as shown in Section 2.4.

(4) Rice planting may pollute and change the environment due to excessive use of pesticides and chemical fertilizers, emission of methane and nitrous oxide, soil gleization of paddy field, soil erosion, and so on. When calculating the comparative benefits between $\mathrm{CK}$ and $\mathrm{CK} 0$, the costs of pesticides and chemical fertilizers reduced in CK because of the ducks' growing and activities in paddy fields are mainly considered and numerically calculated. Of course, there are other forms of contribution and effects of paddy ducks to the comparative benefits, such as the reductive 
emission of greenhouse gases, which are neglected and not analyzed in this paper.

(5) Consider the nearly pollution-free rice product in $\mathrm{CK}$ to be popular and generally more expensive than that is produced by CK0. Assume that the gap between their prices is 1.5 yuan $/ \mathrm{kg}$.

\subsection{Basic Concepts}

Definition 1. Duck density is defined as the ratio between the initial number of the ducklings and the size of the corresponding paddy field. Mathematically,

$$
d=\frac{N_{0}}{A}
$$

where $d$ denotes duck density, $N_{0}$ is the initial number of the ducklings, $A$ is the area of paddy field $\left(\mathrm{hm}^{2}\right)$.

Definition 2. Duck's average survival rate is defined as the ratio between the number of the finally adult live ducks for sale or slaughter and that of the initial stocking ducklings. Mathematically it can be described as

$$
\lambda=\left(\frac{N_{d}}{N_{0}}\right) \times 100 \%,
$$

where $\lambda$ denotes duck average survival rate, $N_{d}$ is the number of the finally adult live ducks for sale or slaughter as food, and $N_{0}$ is the initial number of the ducklings.

$\lambda$ can be stochastically gained or calculated from the related data measured in the given paddy field or in the neighbor district in recent years. And $\lambda$ is one the most important parameters to count and compare the total farming costs and income.

\subsection{Establishment of Models}

2.4.1. Parameters of the Models. Besides the parameters introduced above, including $d, \lambda$, and $A$, the following is used in the established models.

$p, p_{0}$ are average prices of rice product in CK and CK0, respectively. According to Section 2.2, the relationship between $p$ and $p_{0}$ can be expressed as

$$
p=p_{0}+1.5
$$

$x, x_{0}$ are average rice yields per hectare in CK and CK0, respectively.

$P_{N}, P_{P}$, and $P_{K}$ denote the prices of nitrogen, super phosphate, and potassium chloride, respectively.

2.4.2. General Comparative Benefits Model. The farming in CK can generally make better economic and ecological benefits compared with the reference situations in CK0. It consists of the relative increase of production and income, such as the added value of the high-quality rice product, the income from duck stocking, the saving and reduction of the dosage of pesticides, and use of chemical fertilizers, subtracting the extra costs in CK due to duck raising, including the costs of the ducklings, duck feed, nylon net around the border of paddy field, duck sheds, and related manpower of feeding ducks, and keeping them safe and healthy. Therefore, the comparative economic and ecological benefits in CK when there is no special natural constraint from the paddy field and its surroundings based on the assumptions above are mathematically expressed as follows:

$$
\Delta y=\Delta V_{\text {rice }}+V_{\text {duck }}+V_{\text {eco }},
$$

where $\Delta y$ is the comparatively added value; $\Delta V_{\text {rice }}$ denotes the added value in term of the rice product in $C K$, which is generated from higher quality and the larger quantity of rice yields and can be expressed as

$$
\Delta V_{\text {rice }}=\left(p x-p_{0} x_{0}\right) \cdot A,
$$

where $x$ denotes the average yields of rice per hectare and $A$ denotes the area of paddy field $\left(\mathrm{hm}^{2}\right)$.

$V_{\text {duck }}$ denotes net income value of duck raising and is equal to the outcome of adult live ducks subtracting the costs due to duck raising; $V_{\text {eco }}$ denotes the ecological benefits, including two parts, one is produced by duck dung eliminated in the paddy field whose value can be calculated by chemical fertilizers, the other is the value of pesticides reduced in $\mathrm{CK}$ comparing with the situations in CK0.

Furthermore, the three original parts of $\Delta y$ in (4) can be connected closely with duck density $d$ and the complex stocking time $t$. But the basic ranges of $d$ and $t$ ought to be defined first. According to agricultural knowledge, the rice's growth period is generally 110-150 days, and the rational and economic growth period of a typical kind of duck in the middle and lower reaches of Yangzi River is 42-50 days. Considering the main business and major purpose to produce rice, the complex stocking time $t$ in practice is always set to be 50-80 days due to two factors: on the one hand, when it is too short, ducks have little effects on the paddy field and on the grow and yields of rice and little ecological benefit can be attained; on the other hand, if the complex stocking time is too long, it is adverse for rice growing, leading to reduction of rice yields and impacts on the total economic and ecological benefits. That is to say, the range of the variable $t$ is the close interval $[50,80]$, namely, $50 \leq t \leq 80$. After that process, the ducks are collected from the paddy field and transferred to be fattened for sale or slaughter. In addition, the duck density cannot be too large, for too many ducks in the paddy field do not only help to control plant diseases and insert pests in the paddy field, but also tread on the rice plant, which leads to a great reduction of rice yields. In farming practice, the duck density is generally assumed to change from 0 to $600 \mathrm{ducks} / \mathrm{hm}^{2}$, namely, $0<d \leq 600$. Therefore, the discussion next in this paper is based on this premise, namely,

$$
\begin{aligned}
& 0<d \leq 600 \\
& 50 \leq t \leq 80
\end{aligned}
$$

(1) Calculating $\Delta V_{\text {rice }}$ and $V_{\text {duck }}$. Firstly, an experiment, complex cultivating rice and stocking ducks in paddy field 
for 80 days, has been done in the experimental zone of National Rice Research Institute of China to test the effect of duck density on the output of rice paddy and corresponding economic outcome [20]. According to the experimental data and regression analysis, the relation function between rice paddy yields and duck density $d$, when $t$ is 80 days, can be attained; that is,

$$
\left.x(d, t)\right|_{t=80}=\left(-0.0103 d^{2}+2.6314 d+7569.4\right) .
$$

And from the data, the relation function between the net value of ducks $V_{\text {duck }}$ and duck density $d$, when $t$ is equal to 80 days, can be expressed as

$$
\left.V_{\text {duck }}(d, t)\right|_{t=80}=\left(-0.0096 d^{2}+11.3861 d+17.0857\right) \cdot A .
$$

Secondly, based on (7) and (8), the relation function among $x, d$, and $t$ and the relation function among $V_{\text {duck }}, d$, and $t$ can be attained as in the analysis next.

When the complex cultivating and stocking timet is shorter than 80 days, for example, 70 days, the effect of the interaction of ducks and rice on rice yields has not made such a profound sense that the rice yields value must be multiplied by a dynamic coefficient changing with $t$, which can be proximately expressed mathematically as $e^{-(t-80)^{2} / 2 \times 80^{2}}$ based on the analysis of information and data according to related reports [7]. In other words, rice paddy yields $x(d, t)$ can be expressed as follows:

$$
x(d, t)=\left(-0.0103 d^{2}+2.6314 d+7569.4\right) \cdot e^{-(t-80)^{2} / 2 \times 80^{2}} .
$$

On the contrary, when $t$ is shorter than 80 days but longer than 50 days, the net income of ducks is relatively higher than when $t$ is 80 days. When the complex stocking time exceeds 50 days, the ducks are usually more than 65 to 70 days old. But the rational period of duck growth is normally 42 to 50 days. So the ducks demands more food, but without much effect of adding to their weight in this growth stage. They have to be fed 2 or 3 times a day; otherwise, they may do harm to the rice by means of eating some leafs or grain of rice. According to farming experience, it can be supposed that a duck in this growth period consumes $0.1 \mathrm{~kg}$ feed a day by average, which costs man about 0.18 yuan. And the saved feed costs of stocking a duck for $t$ days compared with 80 days, $50 \leq t \leq 80$, are $(80-t) \cdot 0.18$ yuan. Considering duck average survival rate to be $\lambda$, the total saved cost of stocking $A \cdot d$ ducks for $t$ days is assumed to be $(80-t) \cdot 0.18 \cdot \lambda \cdot d \cdot A$. Therefore, if the costs of relative extra manpower to feed them and the small changes of added weight of ducks are neglected, then the net value term $V_{\text {duck }}$ can be attained based on (8) as follows:

$$
\begin{array}{r}
V_{\text {duck }}(d, t)=\left[-0.0096 d^{2}+(11.3861+14.4 \lambda) d\right. \\
-0.18 \cdot \lambda \cdot t \cdot d+17.0857] \cdot A .
\end{array}
$$

Finally, $\Delta V_{\text {rice }}$ is attained according to (3), (5), and (9); that is,

$$
\begin{gathered}
\Delta V_{\text {rice }}=\left[\left(p_{0}+1.5\right)\left(-0.0103 d^{2}+2.6314 d+7569.4\right)\right. \\
\left.\cdot e^{-(t-80)^{2} / 2 \times 80^{2}}-p_{0} x_{0}\right] \cdot A .
\end{gathered}
$$

(2) Calculating $V_{\text {eco }}$. The ecological benefits $V_{\text {eco }}$ consist of two parts, which is described as

$$
V_{\text {eco }}=V_{\text {eco } 1}+V_{\text {eco } 2} \text {, }
$$

where $V_{\text {ecol }}$ denotes the value produced by the duck eliminations in the paddy field, which can be calculated by the value of equivalent chemical fertilizers, and $V_{\mathrm{eco} 2}$ denotes the value of pesticides and herbicides saved and reduced in CK when compared with CK0.

Firstly, according to the reports of the technique of raising ducks in paddy field from China network of poultry disease, the average duck dung eliminated by each duck is about $10 \mathrm{~kg}$ if the duck stocking time in paddy field is not less than 80 days, containing $\mathrm{N} 0.049 \mathrm{~kg}, \mathrm{P}_{2} \mathrm{O}_{5} 0.072 \mathrm{~kg}$, and $\mathrm{K}_{2} \mathrm{O} 0.032 \mathrm{~kg}$, whose value is to be calculated by equivalent chemical fertilizers, such as nitrogen ( $\mathrm{N}$-content percent is $46 \%)$, super phosphate $\left(\mathrm{P}_{2} \mathrm{O}_{5}\right.$-content percent is about $\left.17 \%\right)$, and potassium chloride $\left(\mathrm{K}_{2} \mathrm{O}\right.$-content percent is about $\left.55 \%\right)$. Consider that in the earlier 50 days of complex stocking, when the ducks are in their relative younger growth period, the duck dung eliminated in these 50 days is added up to two fifths of the total number eliminated in 80 days, namely, $10 \mathrm{~kg}$ per duck; that is to say, the average duck dung eliminated by each duck in its earlier complex stocking period of 50 days is assumed to be $4 \mathrm{~kg}$. Given the precondition $50 \leq t \leq 80$, when $t>50$, when the ducks are generally elder than 65 days, it can be supposed that the daily duck elimination reaches its top level in this growth period; that is to say, the average duck dung eliminated by each duck per day in this period is assumed to be $(10-4) / 30 \mathrm{~kg}$. So the relation function among $V_{\text {ecol }}, d$ and $t$ is described as follows:

$$
\begin{aligned}
V_{\mathrm{eco} 1}= & \frac{[4+(6 / 30)(t-50)]}{10} \\
& \cdot\left[\left(\frac{0.049}{46 \%}\right) \times P_{N}+\left(\frac{0.072}{17 \%}\right) \times P_{P}+\left(\frac{0.032}{55 \%}\right) \times P_{K}\right] \\
& \cdot d \cdot \lambda \cdot A,
\end{aligned}
$$

where $P_{N}, P_{P}$, and $P_{K}$ denote the prices of nitrogen, super phosphate, and potassium chloride, respectively.

So (13) can be simplified and expressed as

$$
\begin{aligned}
& V_{\text {ecol }} \\
& =[0.02 t-0.6] \cdot\left[0.107 P_{N}+0.424 P_{P}+0.058 P_{K}\right] \cdot d \cdot \lambda \cdot A .
\end{aligned}
$$

Secondly, the ducks growing in paddy field help to control plant diseases, insert pests, and amount of weeds ecologically. 
So, dosage of pesticides and herbicides are reduced to some extent whose equivalent value term $V_{\text {eco2 }}$ can be numerically exemplified and stochastically calculated during the whole process of rice management. According to the tests and reports [21], if duck density is about 300 ducks $/ \mathrm{hm}^{2}$ and the complex stocking time is between the interval $[60,80]$, the population of weeds in paddy field is so well controlled that there is no need of herbicides any more. Through experiments and tests, it has been observed that it is $100 \%$ effective in the prevention of Chilo suppressalis without using pesticides and the controlling effect on plant diseases and other pests is also obvious. Some researchers estimate that people can save 300 to 400 yuan $/ \mathrm{hm}^{2}$ from the reduced pesticides and herbicides due to the integrated rice-duck farming [22]. But with the growing of rice shoots, the controlling effect of plant diseases and insert pests will be limited. Therefore, if $50 \leq t \leq 80$, the small changes of the disease and insert pests controlling effect due to the changing of $t$ can be neglected; that is to say, $V_{\mathrm{eco} 2}$ is assumed to be expressed with a simple function related only to the variable $d$. According to surveys and observations, the relation function is supposed to be expressed as follows:

$$
\begin{aligned}
& V_{\text {eco2 }} \\
& \quad= \begin{cases}0, & d<15 \\
\left(300 e^{-(d-300)^{2} / 2 \times 100^{2}}-3.327\right) \cdot A, & 15 \leq d \leq 300 \\
\left(\frac{400}{\left(1+e^{-0.036626 d}\right)}-3.327\right) \cdot A, & d>300 .\end{cases}
\end{aligned}
$$

Finally, $V_{\text {eco }}$ can be attained when expressions (12), (14), and (15) are combined.

In a word, given a paddy field, the comparative economic and ecological benefits can be expressed by the relation function of $d$ and $t$, and it can be calculated in the light of (4) with the help of (10)-(15). If an optimal pair of (d, $t$ ) is selected, then the maximal comparative economic and ecological benefits, max $\Delta y$, can be achieved in CK, given other conditions being the same as those in CK0.

\subsubsection{Comparative Benefits Model with Special Constraints.} In Section 2.4.2, there is no consideration of natural constraints, but in farming practice, some rice fields are not completely suitable to establish and keep a rice-duck complex ecosystem due to the handicap or constraints of certain natural or technical factors, such as the assessment of the probability of setting up and keeping or repairing the nylon net around the paddy field, the convenience and costs of irrigating, the difficulties of keeping ducks safe and protecting them against mice and other animals, and the natural demands and related constraints of rice and duck varieties. Subjective constraints of raising ducks in paddy fields have not been considered either. But in practice, there exists some impact of production intentions or political instructions. For example, considering the major business to attain high rice yields to feed the even larger population, some people and governments tend to set their goals of the minimal rice yields when making a decision of selecting a pair of duck density and complex stocking time. The special constraints like that can be reflected and described as the constraints of duck density $d$, complex stocking time $t$, or the relationship between $d$ and $t$ as follows:

$$
f(d) \leq d_{0}, \quad f(d, t)<c, \quad g(d, t)>0 .
$$

Therefore, on the basis of above discussion, an integrated expression is given as follows:

$$
\max \quad \Delta y
$$

$$
\begin{array}{ll}
\text { Subject to, } \quad & f(d) \leq d_{0}, \\
& f(d, t)<c, \\
& g(d, t)>0,
\end{array}
$$

where the calculations of all parts of $\Delta y$ are the same as those in (4), but we now have to test the feasibility of the results according to the constraints expressed in expression (15).

For example, after overall considerations and assessment of all the circumstances about the expected rice's period of growth and natural factors of the paddy field, we come to a conclusion that the given paddy field is partly suitable to establish and keep a rice-duck integrated ecosystem and the constraints of $d$ and $t$ are described as

$$
\begin{gathered}
60 \leq t \leq 80 \\
d \cdot t \leq C \\
d<300
\end{gathered}
$$

where $C$ is a constant, which means that due to some special constraints, the complex stocking time $t$ must be short enough if the farmer want to raise a relative large amount of ducks, or vice versa.

Expression (17) indicates that a sound pair of $(d, t)$ is found and selected so that the max $\Delta y$, maximum comparative economic and ecological benefits, can be achieved under some objective or subjective constraints for integrated riceduck farming in practice.

2.5. Algorithm and Analysis. The models proposed in Section 2.4 reveal the nonlinear relationship between benefits and duck density together with complex stocking time. Some algorithm can be applied to solve the problem, that is, to select an optimal pair of duck density and complex stocking time so that the maximum economic and ecological benefits should be made. Neural Networks do well in nonlinear fitting and have successfully been applied to solve a lot of complex problems in engineering, but a general Neural Network has some defects; for example, its connection weights and thresholds are randomly selected [23, 24]. Considering the Genetic Algorithm to have its advantage of optimization, a hybrid algorithm based on Neural Networks and the Genetic Algorithm is adopted.

2.5.1. Neural Networks (NN). A NN can be logically divided into three layers, namely, the input layer, the hidden layer, 
and the output layer. Each layer consists of certain number of neurons linked by weighted connections to neurons of adjacent layers. $\mathrm{NN}$ can refer to $[25,26]$ in detail.

To apply a NN, it is crucial to make certain of the input and output variables and to set the number of neurons of the input and output layer. Firstly, the two decision variables are found out in this work, namely, duck density $d$ and span $t$ of complex stocking time. So, the number of input neurons is two in this work. Secondly, the number of output neurons is one. The output variable represents the comparative economic and ecological benefits, namely, $\Delta y$. Thirdly, the activation function in the hidden layer is defined as the sigmoid function here.

\subsubsection{Genetic Algorithm (GA)}

(1) Method Representation. In the duck density problem, a vector consisting of the duck density $d$ and complex stocking time $t$, namely, $\mathrm{Q}=\left(d_{i}, t_{i}\right)$, is treated as a chromosome and a decision vector, where the $\left(d_{i}, t_{i}\right)$ is a pair of data with certain constraints like expression (19) that are generated from farming experience and domain knowledge.

(2) Initialization and Coding Process. Randomly generate a chromosome $Q=\left(d_{i}, t_{i}\right)$, and check its feasibility with the given changing ranges and constraints. If it is feasible, it can be accepted as a chromosome. Repeat this process until pop_size initial feasible chromosomes $Q_{1}, Q_{2}, \ldots$, and $Q_{\text {pop_size }}$ are produced. Note that pop_size is the number of the chromosomes.

(3) Selection Process. Assume chromosomes $Q_{1}, Q_{2}, \ldots$, and $Q_{\text {pop_size }}$ to have been rearranged from good to bad according to their objective values. Their adaptability $F_{1}, F_{2}, \ldots$, and $F_{\text {pop_size }}$ is calculated.

The selection process is usually carried out based on a method named spinning the roulette wheel [25]. That is to say, a selection process is completed by means of calculating the fitness of the chromosomes, and the selection probability of an individual chromosome can be calculated as follows:

$$
p_{i}=\frac{f_{i}}{\sum_{i=1}^{p o p s i z e} f_{i}},
$$

where $f_{i}=k F_{i}$ if the target is to find the maximum value, just like the situation in this paper to find the maximum comparative benefit. If $p_{i}>p_{k}$ ( $p_{k}$ is a certain value generated randomly), then chromosome ${ }_{i}$ is selected as a copy. After the selection process, pop_size copies of chromosomes are copied and obtained. Note that pop_size is the number of copies of chromosomes.

(4) Crossover Process. In order to determine the parents for a crossover operation, the following process is executed repeatedly from $i=1$ to pop_size: generating a random number $r$ from the interval $[0,1]$, the chromosome $Q_{i}$ is selected as a parent if $r<$ pcross, where pcross is the probability of crossover. Denote the selected parents by $Q_{1}^{\prime}, Q_{2}^{\prime}, Q_{3}^{\prime}, \ldots$ and divide them into pairs, such as $\left(Q_{1}^{\prime}, Q_{2}^{\prime}\right)$, $\left(Q_{3}^{\prime}, Q_{4}^{\prime}\right)$, and so on. The crossover process on each pair
$\left(Q_{i}^{\prime}, Q_{i+1}^{\prime}\right)$ is illustrated as follows. Generate a random number $\alpha$ from the open interval $(0,1)$; then the crossover operator on $Q_{i}^{\prime}$ and $Q_{i+1}^{\prime}$ will produce two children $Q_{i}^{\prime \prime}$ and $Q_{i+1}^{\prime \prime}$ in the following way:

$$
Q_{i}^{\prime \prime}=\alpha Q_{i}^{\prime}+(1-\alpha) Q_{i+1}^{\prime}, \quad Q_{i+1}^{\prime \prime}=(1-\alpha) Q_{i}^{\prime}+\alpha Q_{i+1}^{\prime} .
$$

If both children satisfy the constraints of the above models, then we replace the parents with them. Otherwise, we keep the feasible one if it exists, and redo the crossover operation by regenerating another real number from $(0,1)$ until two feasible children are obtained.

(5) Mutation Process. In order to determine the parents for a mutation operation, let us do the following process repeatedly from $i=1$ to pop_size: generating a random number $r$ from $[0,1]$. The chromosome $Q_{i}$ is selected as a parent under the condition of $r<$ pmutation, where pmutation is the probability of mutation.

Based on the above ideology, the GA algorithm has usually the following steps [27].

Step 1. Initialize pop_size chromosomes, the probability of crossover pcross, the probability of mutation pmutation, and the maximum generation maxgen.

Step 2. Update the chromosomes by crossover and mutation operations.

Step 3. Calculate the objective value and fitness of all chromosomes.

Step 4. Select the chromosomes by spinning the roulette wheel.

Step 5. Repeat steps 2-4 for a given number of cycles.

Step 6. Report the best fitness and corresponding chromosome as the optimal solution output.

2.6. Duck Density and Complex Stocking Time Selection Algorithm. If Neural Networks (NN) and the Genetic Algorithm (GA) are integrated and optimized, then a hybrid intelligent optimization algorithm named NN-GA can be attained. It is on the basis of the following idea and methodology: to begin with, based on the feature of actual problem of duck density and complex stocking time and related knowledge of $\mathrm{NN}$, an appropriate $\mathrm{NN}$ model together with its parameters is established and its prediction result is reported through the output. Secondly, the prediction output of NN is considered as the fitness value of a chromosome in GA. And then the selection, crossover, and mutation operations of GA are executed accordingly. The algorithm flow does not end until the given maximum generation is reached. Finally, the best fitness and its corresponding chromosome is selected and reported as the optimal solution of the problem to select a suitable pair of duck density and complex stocking time. Its basic steps are as follows [28].

Step 1. Initialize the parameters of NN and GA structures, including the initialization of the number of input, hidden 
and output layers neurons, and the weight vector $w$ in NN, the initialization of pop_size, the probability of crossover pcross, probability of mutation pmutation, and the maximum generation maxgen in GA. Determine the code length of chromosomes in GA.

Step 2. Generate training input-output data of NN based on the practical problem of duck density.

Step 3. Train the NN to approximate the fitness function of the actual problem and save its results.

Step 4. Initialize pop_size chromosomes of GA.

Step 5. Calculate the fitness values for all chromosomes by the trained Neural Network.

Step 6. Select chromosomes by spinning the roulette wheel.

Step 7. Update the chromosomes by crossover and mutation operations.

Step 8. Repeat steps 5-7 for a given number of cycles; namely, repeat maximum generation times.

Step 9. Report the best fitness and the corresponding chromosome as the optimal solution of the practical problem.

The above algorithm has been implemented in the program in MATLAB 2009a.

\section{Results and Discussion}

3.1. Numerical Examples and Results. Considering the problem for a rice-duck complex ecosystem in a paddy field in the suburb of Changsha in China, the rice field is about $1 \mathrm{hm}^{2}$. And its irrigation and other conditions are relatively good for rice-duck integrating farming. The parameters of the NN are given in Section 2.5.1. The parameters of the hybrid algorithm NN-GA are set as follows: the population size pop_size is 20, the maximum number of generations maxgen is 100 , the probability of crossover pcross is 0.4 , and the probability of mutation pmutation is 0.2 . And the parameters of the established models are assumed according to the farming experiences and current market information, which are typically set as the follows: $x_{0}$ is $7642 \mathrm{~kg} / \mathrm{hm}^{2}, p_{0}$ is 2 yuan $/ \mathrm{kg}, \lambda$ is $0.9, P_{N}$ is 2.1 yuan $/ \mathrm{kg}, P_{P}$ is 1.2 yuan $/ \mathrm{kg}$, and $P_{K}$ is 2.95 yuan $/ \mathrm{kg}$.

Example 1. Consider a farmer who wants to establish a riceduck complex ecosystem in his rice paddy field in the suburb of Changsha, which is completely suitable for establishment of a rice-duck complex system. And the minimal $\Delta y$ is set to be 1,000 yuan. After the algorithm is executed, the following results are reported from its output:

$$
\max \Delta y=1.4585 \times 10^{4}, \quad(d, t)=(266,70) .
$$

It denotes that the selection of duck density is 266 ducks $/ \mathrm{hm}^{2}$, the complex cultivating and stocking time span is 70 days, and the maximum value term of comparative economic and ecological benefits is $1.4585 \times 10^{4}$ yuan $/ \mathrm{hm}^{2}$.
Yu et al. [20] have tested the effects of the duck density on rice yields and total economic benefits by means of experiments and analysis. With respect to economic benefits, they suggest the appropriate duck density set in the closed interval [250, 325]. It is consistent with our study. It indicates that the proposed models are feasible.

Example 2. A decision-maker for ecological agriculture hopes to establish a rice-duck complex ecosystem. After analyzing the natural conditions of the rice field, the growing periods and characteristics of certain rice, and duck varieties, he finds that the duck density and complex stocking time problem satisfies the following constraints: $0<d \leq 650$; $d \cdot t \leq 10080 ; 50 \leq t \leq 80$. This problem belongs to the partly suitable situation, which can be solved by the following model:

$$
\begin{array}{ll}
\max & \Delta y \\
\text { Subject to, } & d \cdot t \leq 10080, \\
& 0<d \leq 650, \\
& 50 \leq t \leq 80 .
\end{array}
$$

After the algorithm is executed, the following results can be obtained:

$$
\max \Delta y=1.1938 \times 10^{4} ; \quad(d, t)=(158,64) .
$$

It suggests that he can make the following selection and decision: duck-density $d$ should be set to be 158 ducks $/ \mathrm{hm}^{2}$ and the span $t$ should set to be 64 days. The value of the maximum comparative economic and ecological benefits is $1.1938 \times 10^{4}$ yuan $/ \mathrm{hm}^{2}$.

Further test is made in the light of the proposed models. After initializing the NN structure and the parameters of GA, the following process is experienced. Firstly, select 4000 group of $(d, t, \Delta y)$ satisfying the two conditions $0<d \leq 650$ and $50 \leq t \leq 80$; randomly choose 3900 groups of them as training data, while the other 100 groups as test data of NN. Secondly, treat and use the testing output of the trained NN as the fitness of chromosomes in GA for optimization in terms of the algorithm discussed in Section 2. Note that it is vital to test the feasibility of chromosomes whether they satisfy the constraint of $d \cdot t \leq 10080$. Finally, get output information about the best fitness, namely, the maximum of comparative benefits $\Delta y$, and the corresponding chromosome, namely, the suggested duck density $d$ and stocking time $t$ in the paddy field, after the proposed algorithm has executed. The optimal output of GA is shown as Figure 1.

3.2. Discussion of the Results. It can be seen from Figure 1 that after evolving about 40 generations, the best fitness of each generation, "Bestfitness" in Figure 1, reaches its stable level, that is $1.1938 \times 10^{4}$, which denotes the digital value of the maximum comparative economic and ecological benefits. And after evolving for about 87 generations, the average fitness of each generation, "Avgfitness" in Figure 1, reaches its stable value, which is proximately reaching and coinciding 


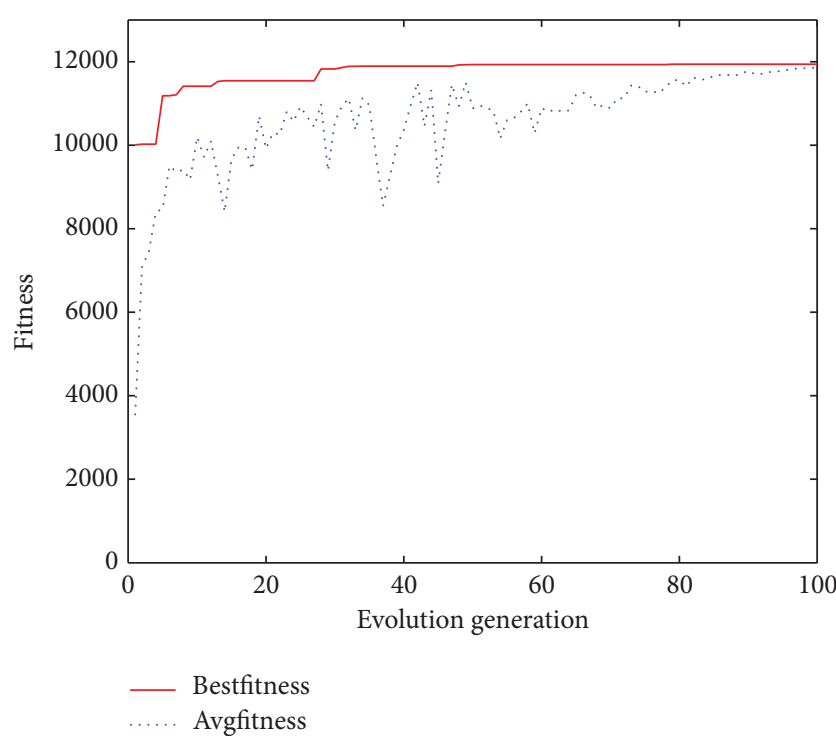

FIGURE 1: Fitness curve of each evolution generation.

with the curve of the best fitness. It means that with evolution, the adaptability and fitness of the individuals are getting better. These reveal that the proposed method is feasible with rational results.

\section{Conclusion}

Rice-duck integrated farming is an effective way and will play an important role in modern bioagriculture, which is characterized with its cleaner and green production and with good ecological effectiveness and benefits. The problem of duck density and stocking time in the rice-duck complex ecosystem is important to promote the development of modern resource-thrift and environment-friendly agriculture, which helps to achieve better economic and ecological benefits. In order to make reasonable suggestions and provide rational reference with respect to this kind of problem, planning models are established on the basis of related domain knowledge, experiments, and exiting researches. Then a hybrid intelligent algorithm, NN-GA, integrating Neural Network (NN) and Genetic Algorithm (GA) is introduced to solve the established models, taking advantage of the nonlinear mapping and fitness ability of $\mathrm{NN}$ and the optimization ability of GA. The tests and examples indicate that the proposed method is feasible. The methodology proposed in this work can also serve as a reference for economic and ecological benefits quantitative analysis and assessment of other green and cleaner agroproduction systems, such as the rice-duckfish or rice-duck-clam complex agroecosystem.

However, there are some limitations with the proposed method. Some data, especially actual data about the effect of complex cultivating and stocking time on economic and ecological benefits, needs to be collected and further analyzed in order to provide better support for the problem. In addition, due to the activities of the paddy ducks, there exists a relative reduction of greenhouse gases emitted from the rice field, which makes ecological benefits too. Thus this ought to be counted in a proper way in future studies. Moreover, their related factors control problems in the riceduck agroecosystem should be considered.

\section{Conflict of Interests}

The authors declare that there is no conflict of interests regarding the publication of this paper.

\section{Acknowledgments}

This work is supported by the Young Scientists Foundation of National Natural Science Foundation of China (Grant no. 61101235), the Planning Subject of "the twelfth five-yearplan" in National Science and Technology of China (Grant no. 2011BAD21B03), and the Major Subject of Science and Technology Foundation of Hunan Province China (Grant no. 2010FJ1006).

\section{References}

[1] F. Di Renzo, R. Bacchetta, L. Sangiorgio, A. Bizzo, and E. Menegola, "The agrochemical fungicide triadimefon induces abnormalities in xenopus laevis embryos," Reproductive Toxicology, vol. 31, no. 4, pp. 486-493, 2011.

[2] M. M. Iqbal and A. M. Goheer, "Greenhouse gas emissions from agro-ecosystems and their contribution to environmental change in the Indus Basin of Pakistan," Advances in Atmospheric Sciences, vol. 25, no. 6, pp. 1043-1052, 2008.

[3] P. Su, X. 1. Liao, Y. Zhang, and H. Huang, "Influencing factors on rice sheath blight epidemics in integrated rice-duck system," Journal of Integrative Agriculture, vol. 11, no. 9, pp. 1462-1473, 2012.

[4] F. Zhiqiang, H. Huang, L. Xiaolan, H. Ying, X. Wei, and H. Baoliang, "Effect of ducks on $\mathrm{CH}_{4}$ emission from paddy soils and its mechanism research in the rice-duck ecosystem," Acta Ecologica Sinica, vol. 28, no. 5, pp. 2107-2114, 2008.

[5] S.-S. Li, S.-H. Wei, R.-L. Zuo, J.-G. Wei, and S. Qiang, "Changes in the weed seed bank over 9 consecutive years of rice-duck farming," Crop Protection, vol. 37, pp. 42-50, 2012.

[6] Z. Qin, J. Zhang, S. Luo, and J. Zhang, "Population control modelling of Cnaphalocrocis medinalis Guenee in rice-duck integrated farming system," Transactions of the Chinese Society of Agricultural Engineering, vol. 26, no. 11, pp. 283-289, 2010 (Chinese).

[7] S. A. Jin, Y. L. Yang, S. B. Gao, Y. Q. Li, C. Y. Jin, and J. Wang, "Effect of raising ducks in paddy field on yield and benefit of rice," Rice in North China, vol. 39, no. 1, pp. 35-37, 2009 (Chinese).

[8] V. V. Krishna, A. G. Drucker, U. Pascual, P. T. Raghu, and E. D. I. O. King, "Estimating compensation payments for onfarm conservation of agricultural biodiversity in developing countries," Ecological Economics, vol. 87, pp. 110-123, 2013.

[9] R. K. Bhushan, "Optimization of cutting parameters for minimizing power consumption and maximizing tool life during machining of $\mathrm{Al}$ alloy $\mathrm{SiC}$ particle composites," Journal of Cleaner Production, vol. 39, pp. 242-254, 2013.

[10] V. A. Balogun and P. T. Mativenga, "Modelling of direct energy requirements in mechanical machining processes," Journal of Cleaner Production, vol. 41, pp. 179-186, 2013. 
[11] S. He and F. Liu, "Robust stabilization of stochastic Markovian jumping systems via proportional-integral control," Signal Processing, vol. 91, no. 11, pp. 2478-2486, 2011.

[12] S. He and F. Liu, "Robust peak-to-peak filtering for Markov jump systems," Signal Processing, vol. 90, no. 2, pp. 513-522, 2010.

[13] J. Kim, M. J. Realff, and J. H. Lee, "Optimal design and global sensitivity analysis of biomass supply chain networks for biofuels under uncertainty," Computers and Chemical Engineering, vol. 35, no. 9, pp. 1738-1751, 2011.

[14] D. El Chami, A. Scardigno, and G. Malorgio, "Impacts of combined technical and economic measures on water saving in agriculture under water availability uncertainty," Water Resources Management, vol. 25, no. 14, pp. 3911-3929, 2011.

[15] X. L. Sun, S. C. Wu, H. L. Wang et al., "Uncertainty analysis for the evaluation of agricultural soil quality based on digital soil maps," Soil Science Society of America Journal, vol. 76, no. 4, pp. 1379-1389, 2012.

[16] S. Peña-Haro, M. Pulido-Velazquez, and C. Llopis-Albert, "Stochastic hydro-economic modeling for optimal management of agricultural groundwater nitrate pollution under hydraulic conductivity uncertainty," Environmental Modelling and Software, vol. 26, no. 8, pp. 999-1008, 2011.

[17] G. Tian, Y. Liu, Q. Tian, and J. Chu, "Evaluation model and algorithm of product disassembly process with stochastic feature," Clean Technologies and Environmental Policy, vol. 14, no. 2, pp. 345-356, 2012.

[18] G. Tian, J. Chu, Y. Liu, H. Ke, X. Zhao, and G. Xu, "Expected energy analysis for industrial process planning problem with fuzzy time parameters," Computers and Chemical Engineering, vol. 35, no. 12, pp. 2905-2912, 2011.

[19] V. Özkir and H. Bashgil, "Multi-objective optimization of closed-loop supply chains in uncertain environment," Journal of Cleaner Production, vol. 41, pp. 114-125, 2013.

[20] S. M. Yu, Q. Y. Jin, L. F. Zhu, Y. N. Ouyang, and D. H. Xu, "Effects of duck density on rice yields and economic benefits," Zhejiang Agrosci, no. 1, pp. 68-71, 2008 (Chinese).

[21] P.-A. Xiang, H. Huang, D.-X. Gan, and M. Huang, "An environmental economics analysis of a rice-duck ecological management technique with no-tillage," Acta Ecologica Sinica, vol. 25, no. 8, pp. 1981-1986, 2005.

[22] T. H. Yan, "Effect of rice-duck complex management on controlling rice diseases and insert pests and promotion of income," Science \& Technology Information, vol. 27, pp. 377-378, 2011 (Chinese).

[23] Y. J. Ji, R. J. Jiao, L. Chen, and C. L. Wu, "Green modular design for material efficiency: a leader-follower joint optimization model," Journal of Cleaner Production, vol. 41, pp. 187-201, 2013.

[24] B. Liu and K. Iwamura, "Modelling stochastic decision systems using dependent-chance programming," European Journal of Operational Research, vol. 101, no. 1, pp. 193-203, 1997.

[25] G. Tian, Y. Liu, H. Zhang, J. Chu, and G. Xu, "Chance programming models for time-energy trade-off problem of product disassembly process with multiple stochastic variables," Advanced Science Letters, vol. 4, no. 4-5, pp. 1851-1854, 2011.

[26] G. D. Tian, M. C. Zhou, and J .W. Chu, "A chance constrained programming approach to determine optimal disassembly sequence," IEEE Transactions on Automation Science and Engineering, vol. 10, no. 4, pp. 1004-1013, 2013.

[27] G. D. Tian, J. W. Chu, and T. G. Qiang, "Influence factor analysis and prediction models for component removal time in manufacturing," Proceedings of the Institution of Mechanical Engineers B, vol. 227, no. 10, pp. 1533-1540, 2013.

[28] G. D. Tian, T. G. Qiang, J. W. Chu, G. Xu, and W. Zhou, "Efficiency optimization for disassembly tools via using NNGA approach," Mathematical Problems in Engineering, vol. 2013, Article ID 173736, 8 pages, 2013. 


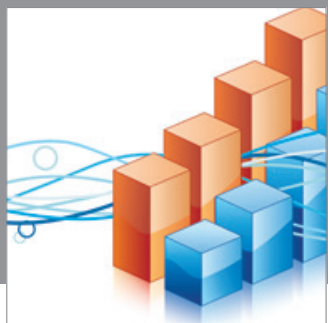

Advances in

Operations Research

mansans

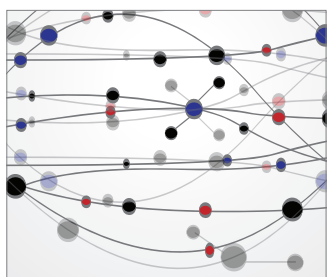

The Scientific World Journal
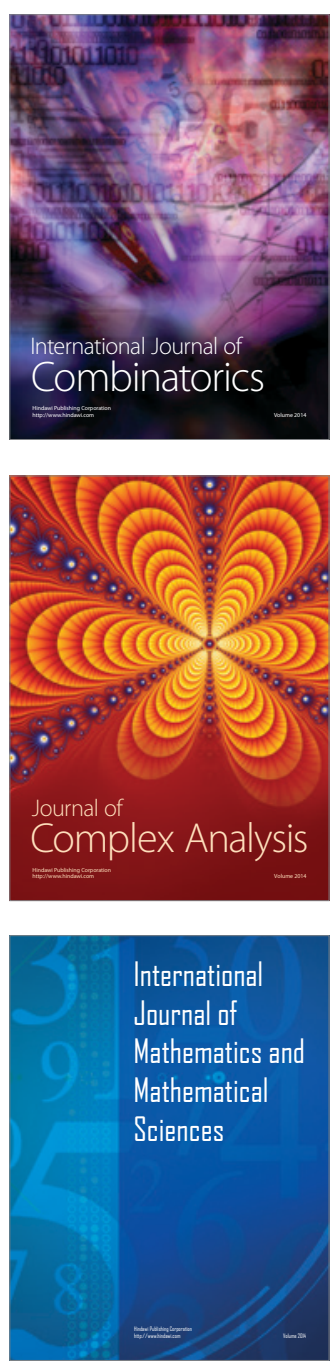
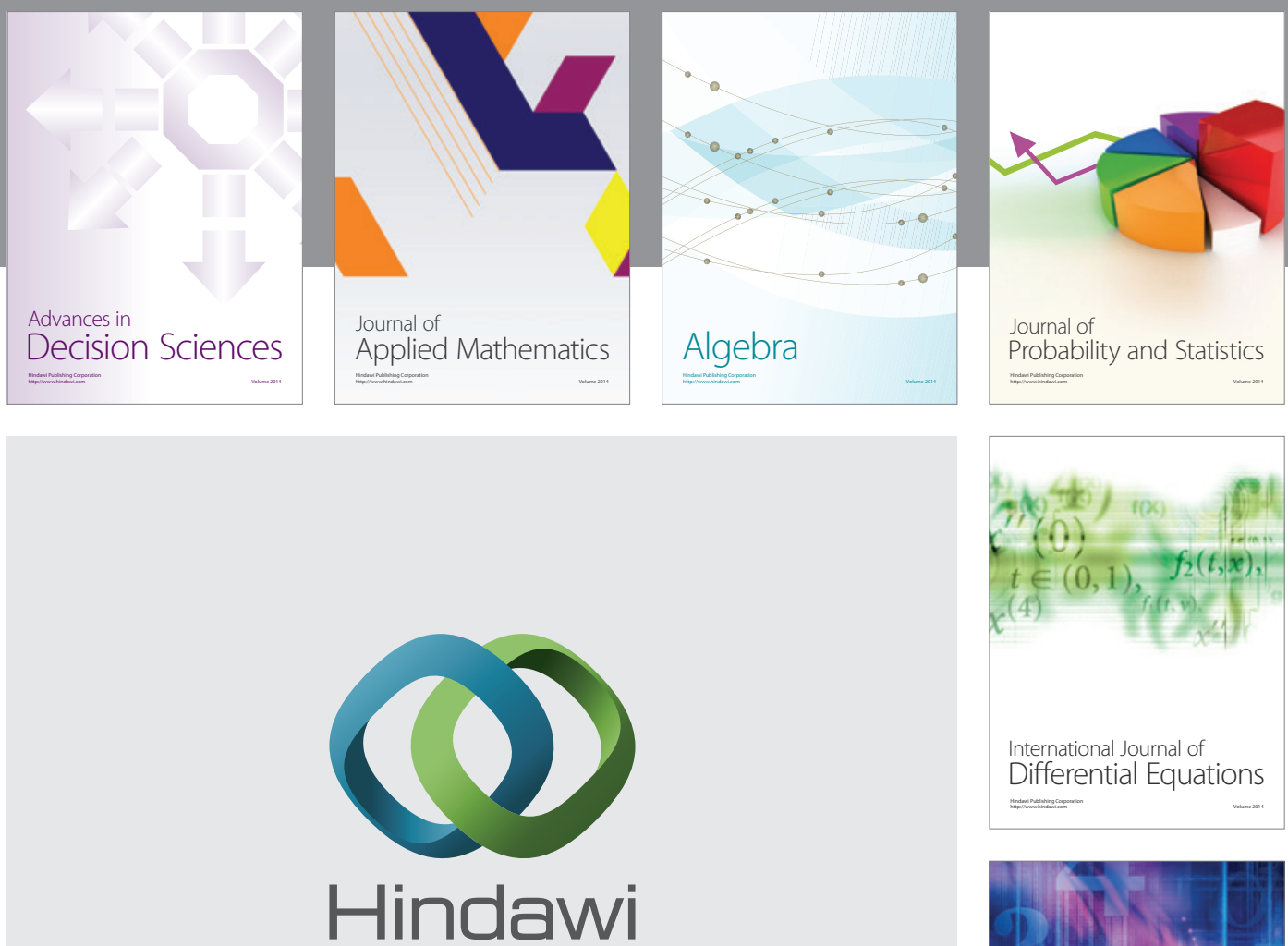

Submit your manuscripts at http://www.hindawi.com
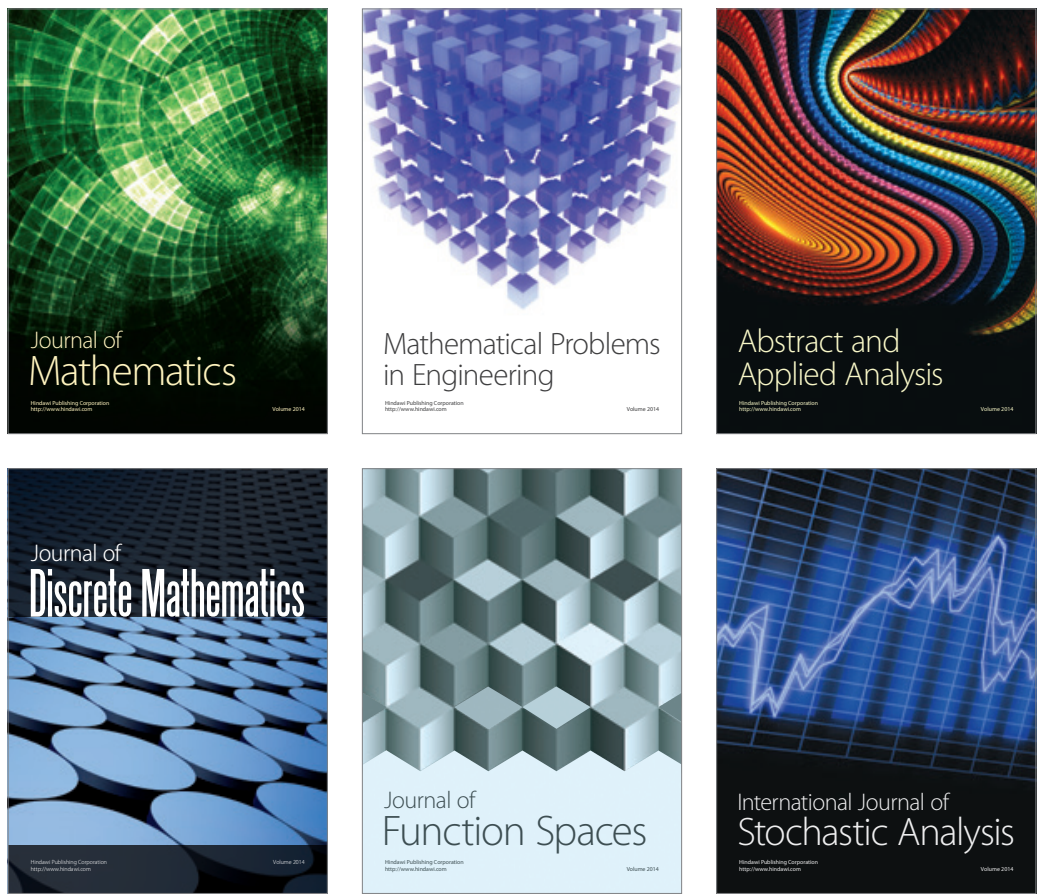

Journal of

Function Spaces

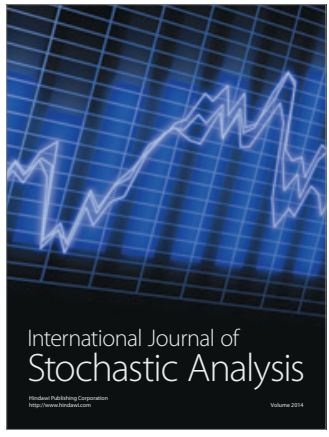

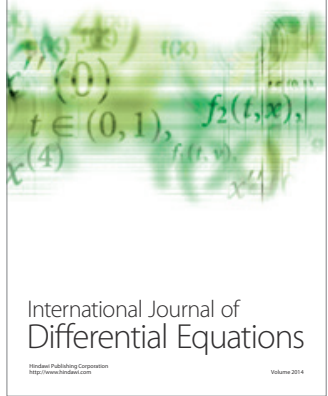
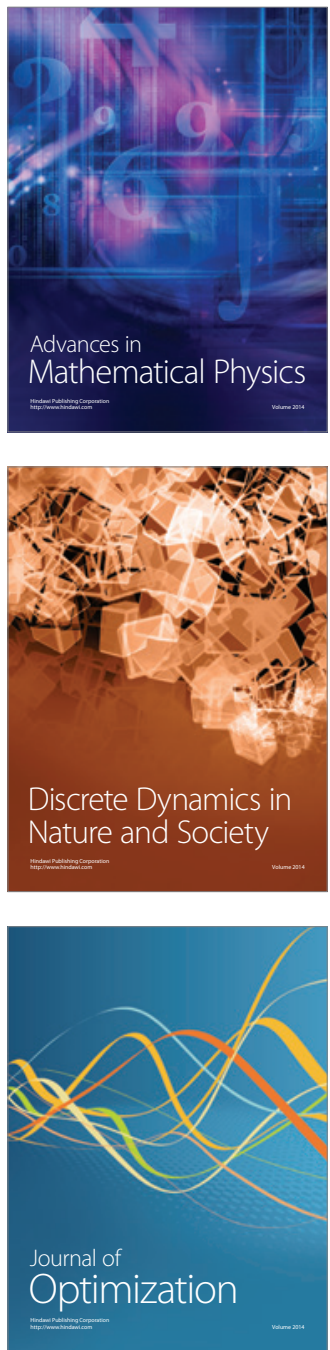